\title{
The Influence of X-Factor (Trunk Rotation) and Experience on the Quality of the Badminton Forehand Smash
}

\author{
by \\ Zhao Zhang1, Shiming Lit,2, Bingjun Wan ${ }^{1,3}$, Peter Visentin ${ }^{4}$, Qinxian Jiang ${ }^{1,5}$, \\ Mary Dyck', Hua Li ${ }^{6}$, Gongbing Shan ${ }^{1,3,7}$
}

No existing studies of badminton technique have used full-body biomechanical modeling based on threedimensional (3D) motion capture to quantify the kinematics of the sport. The purposes of the current study were to: 1) quantitatively describe kinematic characteristics of the forehand smash using a 15-segment, full-body biomechanical model, 2) examine and compare kinematic differences between novice and skilled players with a focus on trunk rotation (the X-factor), and 3) through this comparison, identify principal parameters that contributed to the quality of the skill. Together, these findings have the potential to assist coaches and players in the teaching and learning of the forehand smash. Twenty-four participants were divided into two groups (novice, $n=10$ and skilled, $n=14$ ). A 10-camera VICON MX40 motion capture system (200 frames/s) was used to quantify full-body kinematics, racket movement and the flight of the shuttlecock. Results confirmed that skilled players utilized more trunk rotation than novices. In two ways, trunk rotation (the X-factor) was shown to be vital for maximizing the release speed of the shuttlecock - an important measure of the quality of the forehand smash. First, more trunk rotation invoked greater lengthening in the pectoralis major (PM) during the preparation phase of the stroke which helped generate an explosive muscle contraction. Second, larger range of motion (ROM) induced by trunk rotation facilitated a whip-like (proximal to distal) control sequence among the body segments responsible for increasing racket speed. These results suggest that training intended to increase the efficacy of this skill needs to focus on how the X-factor is incorporated into the kinematic chain of the arm and the racket.

Key words: 3D motion capture, full-body modeling, kinematics, whip-like movement.

\section{Introduction}

The popularity of badminton as a sport is rapidly increasing (Brundle, 1963; El-Gizawy and Akl, 2014; Wang and Moffit, 2009). After its Olympic debut at the 1992 Barcelona Summer Games, badminton attracted world-wide attention (Ming, 1993). Since its rules are straightforward, the equipment required to participate can be low cost and it can be played in a relatively small area, badminton can easily appeal to individuals of varying ages, physical abilities and socioeconomic condition.

The forehand smash is an important offensive technique in badminton (El-Gizawy and Akl, 2014; Gowitzke and Waddell, 1991). In the

\footnotetext{
1 - Department of Kinesiology, University of Lethbridge, Canada.

2 - Department of Physical Education, Ocean University of China (Qingdao), China.

3 - School of Physical Education, Shaanxi Normal University (Xian), China.

4 - Faculty of Fine Art, University of Lethbridge, Canada.

5 - Key Laboratory of Exercise and Health Sciences of the Ministry of Education, Shanghai University of Sport, China.

6 - Department of Math \& Computer Science, University of Lethbridge, Canada.

7 - Department of Physical Education, Xizhou Teachers' University (Shanxi), China.
} 
stroke, the shuttle is hit toward the opponent's court with high speed and at a steep downward angle (Yap, 2012). The world's fastest recorded badminton smash was $332 \mathrm{~km} / \mathrm{h}$ (206 mph) hit by Chinese star Haifeng Fu in the Sudirman Cup in 2005 (Xinhua News, 2005). Due to the high speeds generated by the stroke, the forehand smash is a shot that frequently determines winning of points during a game (El-Gizawy and Akl, 2014; Osiński, 2003). Thus, from a competitive sports perspective, the smash deserves further scientific research (Brundle, 1963; Sakurai and Ohtsuki, 2000). Biomechanical analysis is an ideal methodology to accomplish this.

The forehand smash is commonly divided into three phases for analytic and instructional purposes (Figure 1): preparation, acceleration and follow through (Brahms, 2014; Yap, 2012).

In the preparation phase, a player adjusts for the shift in the center of gravity that is caused by the movement of the racket arm; the racketside leg supports this shift by stepping in a posterior, and slightly lateral direction. The right elbow is moved in posterior and lateral directions (primarily by shoulder extension and abduction), and the wrist is extended causing the racket-head (RH) to point upward. To balance the posture and improve aiming, pointing the free (non-racket) hand toward the shuttlecock is a common instructional directive. The acceleration phase of the stroke consists of a backswing followed by a forward swing. These two components are delineated by the $\mathrm{RH}$ reaching its lowest point at the end of the backswing. After this, the RH moves forward until the point of impact with the shuttlecock, where the follow-through phase begins. Two factors dominate maximization of shuttlecock speeds during the forehand smash: 1) the forward swing speed determined by the angular velocity of the arm-racket kinematic chain, and 2) the length of that kinematic chain. From a fundamental physics standpoint, at any given angular velocity, a fully extended (longer) arm will generate higher shuttlecock velocities than a bent (shorter) arm. Thus, players are typically directed to make contact with the shuttlecock with arm fully extended (the elbow should be straightened on contact with the shuttle), as high as possible on the racket, and slightly in front of the body to control the shuttlecock's flight (downward) direction. A fast flexion (increased angular velocity) of the wrist is invoked immediately before the point of impact, providing the stroke with extra power (racket velocity) and helping to control the downward flight direction. Finally, a follow-through phase is required to dissipate excess momentum after impact. This can be accomplished by pulling down the racket-arm and crossing it toward the non-racket side, while shifting body weight from the rear foot to the front foot.

Power is a primary key to the final quality of the smash. Li et al (2016) as well as Lo and Stark (1991) confirmed that power and speed are the major factors making the forehand smash a powerful offensive weapon. Unfortunately, the scientific understanding of the forehand smash continues to lag behind its practice as most participants acquire the skills by learning from individual experience rather than through research-based instruction. To date, most forehand smash research is concentrated on the upper limbs such as the shoulders, elbows and wrists in order to discover and understand the kinematic and kinetic characteristics, as well as the relationship between joint movement and smash performance.

Rantzmayer (1977) initiated the scientific discussion on power strokes with focus on a "wrist snap" technique. Tang et al. (1995) used a specialized marker system and analysis method to suggest that the increased supination of the forearm just before its rapid pronation constituted a stretch-shortening cycle (SSC), which served to enhance the speed of the movement. Subsequently, much of the research regarding the radio-ulna joint movement of a badminton smash has been conducted using cinematography and/or biomechanical analysis, with similar results to the above conclusion (Phomsoupha and Laffaye, 2015; Li et al, 2016). However, empirical data regarding trunk movement is hardly addressed in existing badminton research and, as such, the understanding of joint coordination and motor control in relation to smashing quality remains incomplete. Hence, the trunk segment should be considered and analyzed as part of establishing the qualities governing the forehand smash.

Evidence to support this assertion can be found in studies of other sports skills, such as the golf swing, the baseball pitch, the tennis serve and the volleyball spike. Studies that include 
consideration of trunk movement conclude that it contributes significantly to the efficacy of these techniques (Adrian and Enberg, 1971; Elliott et al., 1989; Werner et al., 2008; Zhang and Shan, 2014). In golf, researchers have identified professional golfers to have larger and faster trunk rotation than amateurs (Chu et al., 2010; Shan et al., 2011; Zhang and Shan, 2014). Trunk rotation, the relative rotation of the shoulders with respect to the hips (the angle between a line connecting the anterior superior iliac crests of the pelvic and a line connecting the acromion markers in the upper torso area), has become known in golf research as the "X-Factor" (Cheetham et al., 2001). Mcteige et al. (1994) concluded that a large XFactor at the top of a golf backswing was a key parameter in generating greater club head velocity at impact.

In baseball pitching, Werner et al. (2008) indicated that, for a typical throwing skill, the trunk segment played a vital role in: 1) transferring the power generated in the lower extremities up through the arm, 2) stabilizing the body, and 3) allowing the arm to undergo a whiplike movement to generate velocity. Seroyer et al. (2010) reported large variability in ball velocity found among baseball pitchers to be related to their use of different amounts of transverse trunk rotation. Still other studies confirm that, during the pitching process, the contribution of trunk rotation to total body momentum and the final ball speed was significant in the primary plane of movement (Aguinaldo et al., 2007; Dapena, 1978; Putnam, 1993). In summary, trunk rotation appears to play a major role in transferring power from the lower extremities to the upper extremities, in creating larger effective range of motion (ROM) in the throwing action and in generating high-velocity ball release.

In tennis, almost all strokes can be characterized by trunk and shoulder rotation. From the preparatory position to the completion of the backswing, the trunk and shoulder experience large ROM (approximately 120o) (Elliott, 2000; Elliott et al., 1989). For the forehand groundstroke, Ellenbecker and Roetert (2004) reported the hip-line to shoulder-line angle difference (i.e. the X-Factor) to be approximately 30o for skilled players.

In volleyball research, Adrian and Enberg (1971) reported the sequence of movements in the spike to be similar to that of both the badminton forehand smash and the tennis serve: trunk rotation followed by upper arm, then forearm and hand movement.

In summary, trunk rotation is integral to the generation of power in many sports. It facilitates transfer of energy through the kinetic chain of the body from the lower to the upper extremities. Previous research confirms the importance of the "X-Factor" for golf, baseball, tennis and volleyball during the golf swing, the baseball pitch, the tennis serve and the volleyball spike (Ellenbecker and Davies, 2001; Roetert and Groppel, 2001). It is not unreasonable to postulate that the X-Factor may play a role in many different sports activities. No studies to date have examined the X-Factor in the sport of badminton. Hence, the current study aimed to examine the role of the X-Factor in the badminton forehand smash. The purposes of the study were to: 1) quantitatively determine kinematic characteristics of the forehand smash using a 15-segment, fullbody biomechanical model (Shan et al., 2015; Shan and Westerhoff, 2005; Zhang and Shan, 2014), 2) examine and compare kinematic differences between novice and skilled players with a focus on trunk rotation (the X-factor), and 3) through this comparison, identify principal parameters that contributed to the quality of the skill. It is hoped that this new information can assist coaches in the creation of goal-oriented drills which speed up the learning process at the earliest stages of skill acquisition.

\section{Material and Methods}

\section{Subjects}

A total of 24 participants (22 righthanded, 2 left-handed, age 20-35) were measured (male: $\mathrm{n}=17$; female: $\mathrm{n}=7$ ). The skilled group $(S G, n=14)$ required a minimum of four years' experience in a competitive badminton training environment ( $6.6 \pm 3.1$ years). The novice group $(\mathrm{NG}, \mathrm{n}=10)$ did not have any formal training after the secondary school (0 years). The SG and NG were similar in age $(23.2 \pm 2.8$ years vs. $24.3 \pm$ 4.7), body height $(1.77 \pm 0.05 \mathrm{~m}$ vs. $1.71 \pm 0.07 \mathrm{~m})$, and body mass $(71.56 \pm 7.73 \mathrm{~kg}$ vs. $62.05 \pm 9.24 \mathrm{~kg})$. Ethics approval was obtained from the University of Lethbridge (Canada) and all participants completed informed consent documents prior to data collection. 


\section{D Motion capture and laboratory set-up}

A 10-camera VICON MX40 motion capture system (VICON Motion Systems, Oxford Metrics Ltd., Oxford, England, www.vicon.com) was set up to capture movement and track markers ( $9 \mathrm{~mm}$ in diameter) at a rate of 200 frames/s. Calibration residuals were determined in accordance with VICON's guidelines and yielded positional data accurate within $1 \mathrm{~mm}$. It is worthy to mention that motion capture technology permits considerable freedom of movement for the participant which allows them to maintain their "natural" motor behaviors, thus, the measurement process has negligible influence on participants' personal playing styles. The effective 3D motion capture volume was $6.7 \mathrm{~m}$ in length, $6.0 \mathrm{~m}$ in width and $3.0 \mathrm{~m}$ in height (the standard size of a playing side). A net was set at $1.55 \mathrm{~m}$ (standard net height). Together these conditions mimicked that of a playing environment.

The same YONEX ARCSABER 001 series racket (weight: $85-89 \mathrm{~g}$, length: $684 \mathrm{~mm}$, material: graphite) and a standard YONEX shuttlecock (weight: 4.74-5.50 g) were used for all trials. Sixteen reflective adhesive markers were placed on the equipment as follows: twelve on the racket (one on the handle base; three taped on the shaft and eight on the head), one on the shuttle (taped on the cork) and three on the net. Thirty-nine spherical, reflective markers (diameter $=9 \mathrm{~mm}$ ) were placed on each participant at the standard anatomic landmarks necessary to create a 15 segment, full-body biomechanical model (Shan and Bohn, 2003; Shan et al., 2004; Shan and Westerhoff, 2005). The placement of body markers was as follows: four markers on the head (one each on the left and right temples and two on the posterior portion of the parietal bone); five markers on the upper trunk (one each on the sternal notch, xiphoid process, C7 and T10 vertebrae and the right back); seven markers on each upper limb (acromion processes, lateral epicondyles of the humerus, styloid processes of the ulna and radius, third metacarpophalangeal joints, and upper and lower arms); four markers on the pelvis (one each on left and right anterior and posterior superior iliac crests); and six markers on each lower limb (upper thigh, lateral condyle of the tibia, lateral side of the tibia, lateral malleolus of the fibula, calcaneal tuberosity, head of halluces).

Raw 3D data was smoothed using a fivepoint moving average filter (weighted: 1-3-4-3-1). This supplied kinematic information such as marker positions, positional changes, velocities and accelerations. A 15-segment biomechanical body model was constructed using this data. Data from equipment markers allowed the modeling of the racket, shuttlecock and net. Using the fundamental precepts of physics, simple positional data from consecutive frames of reconstructed data was translated into movements of the forehand smash (Figure 1). In 3D body modeling, inertial characteristics for each segment were determined by applying anthropometric regression equations with individual body mass, body height and gender as input parameters (Shan and Bohn, 2003). Using our biomechanical model (racket, shuttlecock and full body), quality parameters and movement parameters (please see the definition of parameters below) of the forehand smash were quantified.

\section{Experimental procedure}

The experimental procedure was as follows for each participant: 1) markers were placed on the participant and the system was calibrated to the subject; 2) the participant was given time to warm up in a manner he/she deemed appropriate, in order to become familiarized with the testing environment; 3) trial data was gathered. For each participant, five forehand smashes were recorded. Three forehand smashes, those generating the highest shuttle flight velocities, were selected for data analysis. A shuttlecock was hung vertically from the ceiling of the laboratory, $4 \mathrm{~m}$ from the net. To compensate for anthropometric variation, each participant determined the height of the shuttlecock according to his/her personal preference. To minimize compounding variables, no jumping was permitted.

\section{The definition of selected parameters}

Quality Parameters:

1. Shuttlecock Maximum Flight Velocity $\left(V_{\max }\right)$

The maximum flight velocity of the shuttlecock directly reflects athletes' technical proficiency as, when a shuttlecock travels at high velocity, it will minimize the available reaction time for a successful return volley. $V_{\max }$ was determined by frame-to-frame analysis of the 
motion capture data pertaining to the shuttlecock. 2. Past-Net Height $\left(\mathrm{H}_{\mathrm{p}-\mathrm{n}}\right)$ (Figure 2)

The $\mathrm{H}_{\mathrm{p}-\mathrm{n}}$ was the vertical distance between the shuttlecock and the net, as the shuttlecock passed the net. A forehand smash typically directs the shuttlecock in a steep downward trajectory, both to minimize the time that an opponent has to return the volley and to ensure that the shuttlecock stays within the boundaries of the game-play. The $\mathrm{H}_{\mathrm{p}-\mathrm{n}}$ is thus a way to evaluate the quality of a forehand smash; a good smash has a shorter $\mathrm{H}_{\mathrm{p} \text {-n. }}$. The $\mathrm{H}_{\mathrm{p}-\mathrm{n}}$ was obtained from 3D motion capture data.

3. Shuttlecock Flight Angle (Ashuttle) (Figure 2)

The shuttlecock flight angle was defined as the angle between the direction of the shuttlecock flight and the horizontal plane immediately after impact from the forehand smash (Figure 2). Ashuttle is a parameter that affects flight distance and flight time. Ashuttle and $\mathrm{H}_{\mathrm{p}-\mathrm{n}}$ are complementary. The $3 \mathrm{D}$ motion capture process establishes an XYZ coordinate system from which this parameter can be determined.

\section{Movement Parameters}

Biomechanical modeling permits the calculation of a variety of parameters related to body movement. Through the 3D capture of anatomic landmarks and the frame to frame position changes of these landmarks, an individualized skeletal model was constructed for each subject. Using anatomic norms, muscles and other soft tissue can be attached to the skeleton at typical attachment points. This allows the calculation of joint angles and muscle lengths as well as changes in these over the course of the trial.

1. Trunk Rotation (X-factor) (Figure 2)

The $\mathrm{X}$-factor is defined as the angle between the line connecting the anterior superior iliac crests of the pelvic bone and a line connecting the right and left acromion in the upper torso area. The X-factor was calculated in the manner described by Meister et al. (2011) by projecting these two lines onto the horizontal plane.

2. ROM

The movement of each joint reflects players' ROM, and is a measure of both capability and training. The biomechanical model employed allowed the quantification of both maximum and minimum joint angles during each trial. ROM was calculated as the difference of these. ROM for each of the following was determined: 1) shoulder (flexion/extension, abduction/adduction, and rotation), 2) elbow (flexion /extension), and 3) wrist (flexion/extension).

3. Lengthening of Pectoralis Major

The pectoralis major (PM) is a muscle that contributes to the movement of the shoulder joint. It has three primary actions in this regard: 1) flexion of the humerus (e.g. throwing a ball sidearm); 2) adduction of the humerus (e.g. flapping the arms); 3) rotation of the humerus (e.g. armwrestling) (Hamilton et al., 2002). PM lengthening can deeply reflect the movement of the shoulder joint from the respect of muscular constriction. The biomechanical model calculated muscle lengths by considering the distance between normal anatomical attachment points of the muscles on a human skeleton. As the skeleton moved, dynamic lengthening and shortening were determined.

\section{Data processing and Statistical Analysis}

Filtered data was used as input for the 15segment biomechanical model. All parameters obtained from the VICON motion capture system and biomechanical modeling were analyzed using SPSS Statistics v.22.0 (SPSS Inc., Chicago, IL). Results are presented using descriptive statistics. Further, independent $t$-tests were applied to contrast differences between novice and skilled players on both smash quality and movement parameters. Statistical significance was defined as $p<0.05$. Indications of an 'increase' in a parameter (percentage change) were calculated using the following formula [(large value-small value)/small value]. This formula was also used for determining the percentage differences between groups.

Correlations were computed within subjects between $\mathrm{X}$-factor and $\mathrm{V}_{\max }, \mathrm{X}$-factor and

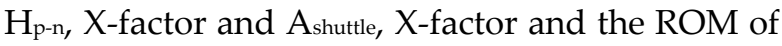
the shoulder (flexion/extension, abduction/adduction, and twist), elbow (flexion/extension) and wrist (flexion/extension), and $\mathrm{X}$-factor and PM using the Pearson correlation coefficient (Lawrence and Lin, 1989; Stigler, 1989). The results of correlation analyses were used to identify factors related to the quality of the smash.

\section{Results}

The purposes of this study were to: 1) quantitatively determine kinematic characteristics 
of the forehand smash using a 15-segment, fullbody biomechanical model, 2) examine and compare kinematic differences between novice and skilled players with a focus on trunk rotation (the $\mathrm{X}$-factor), and 3) through this comparison, identify principal parameters that contributed to the quality of the skill.

Three-dimensional motion analysis of selected parameters revealed distinguishable kinematic characteristics (mean \pm SD) and significant differences between SG and NG (Tables 1 and 2). Based on the power curves for comparisons, standard deviation and the significance level (Dixon and Massey, 1969; Wilkinson and Strkalj, 2005) for the sample size, the differences found in this study have more than $90 \%$ statistic power.

Comparing the SG to the NG, a highly significant difference $(p<0.01)$ was observed in each $\mathrm{V}_{\max }, \mathrm{H}_{\mathrm{p}-\mathrm{n} \text {, }}$ and Ashuttle (Table 1). The $\mathrm{V}_{\max }$ of the SG $(45.31 \pm 7.81 \mathrm{~m} / \mathrm{s})$ was over $30 \%$ higher than that of the NG $(34.64 \pm 8.80 \mathrm{~m} / \mathrm{s})$. The $\mathrm{H}_{\mathrm{p}-\mathrm{n}}$ of the NG $(0.49 \pm 0.25)$ was more than six times higher than that of the SG $(0.08 \pm 0.49)$. The Ashuttle of the SG $\left(14.8 \pm 8.0^{\circ}\right)$ showed a downward trajectory that was four times steeper than that of the NG $\left(3.7 \pm 5.2^{\circ}\right)$.

There was a highly significant difference $(p<0.01)$ in the X-factor between the SG $(46.9 \pm$ $\left.11.2^{\circ}\right)$ and the NG $\left(36.7 \pm 8.2^{\circ}\right)$, a $28 \%$ difference. For shoulder ROM, a highly significant difference existed only for rotation. For the SG (107.5 \pm $\left.30.9^{\circ}\right)$, rotation was more than twice than that of the NG $\left(47.7 \pm 20.4^{\circ}\right)$. Statistical tests showed no significant differences between the SG and NG for flexion/extension and abduction/adduction ROM of the shoulder $(p=0.58$ and $p=0.21$, respectively). Highly significant differences $(p<$ 0.01 ) also existed for flexion/extension ROM in the elbow and wrist flexion. The SG had $30 \%$ more $\left(70.6 \pm 9.1^{\circ}\right.$ vs $\left.54.0 \pm 22.9^{\circ}\right)$ ) elbow flexion/extension and $170 \%$ more $\left(85.9 \pm 50.4^{\circ}\right.$ vs $\left.31.7 \pm 23.7^{\circ}\right)$ wrist flexion/extension than the NG. PM is expressed as a percentage change in length from a resting state (Table 2). For the SG $(40.3 \pm 10.9 \%)$, PM lengthening was $38 \%$ more than for the NG $(29.2 \pm$ $10.9 \%)(p<0.01)$.

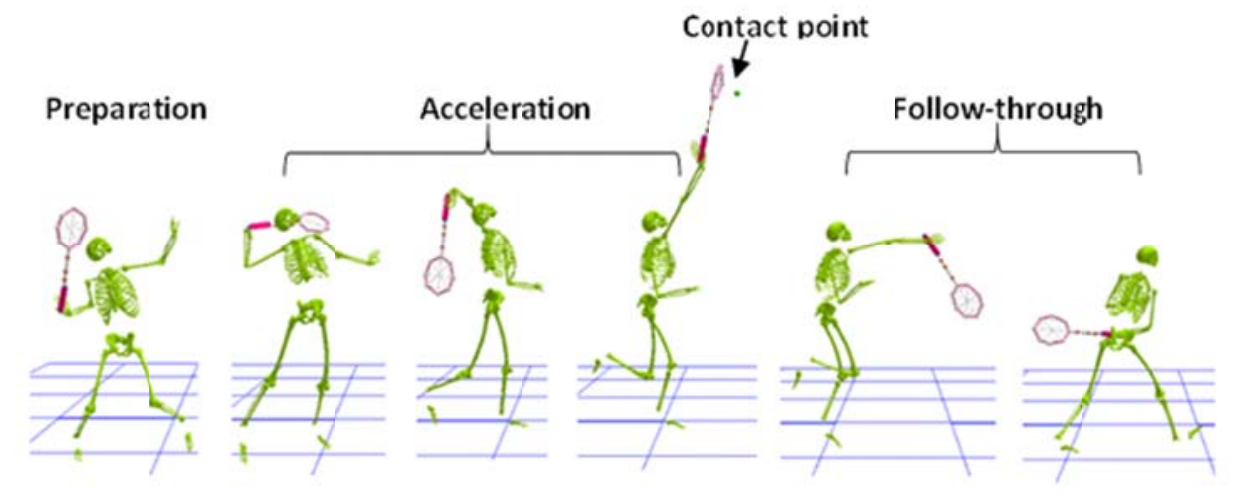

Figure 1

Phases of the badminton smash for a right-handed player 

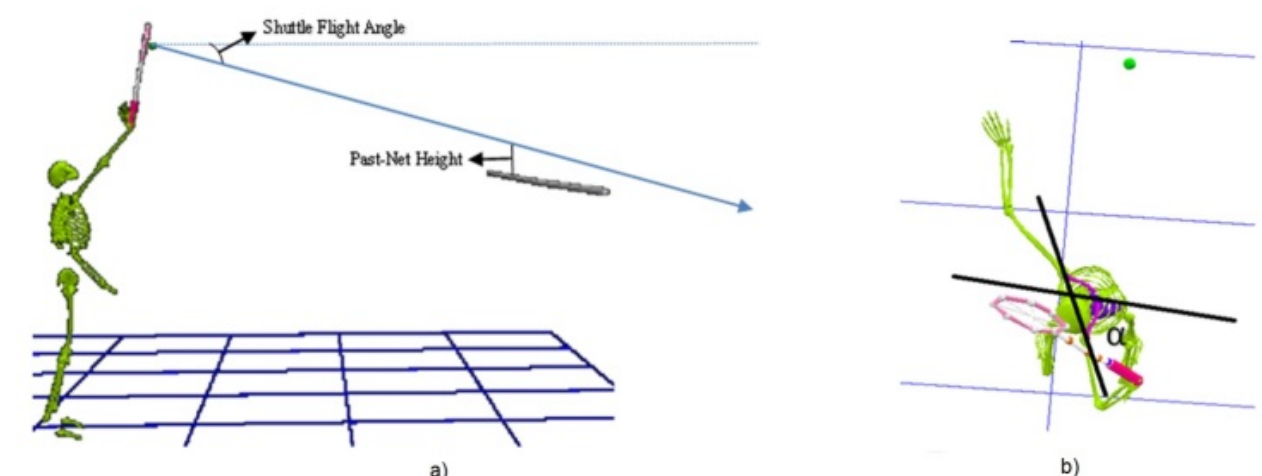

b)

Figure 2

a) Shuttle flight angle (Ashuttle $)$ and past-net height $\left(H_{p-n}\right)$.

b) X-factor: the angle $(\alpha)$ of trunk rotation.

Table 1

Kinematic Data of Smash Quality Parameters

\begin{tabular}{ccccc}
\hline & SG & NG & $p$ & t \\
\hline $\mathrm{V}_{\max }(\mathrm{m} / \mathrm{s})$ & $45.31 \pm 7.81$ & $34.64 \pm 8.88$ & $* *$ & 5.27 \\
$\mathrm{H}_{\mathrm{p}-\mathrm{n}}(\mathrm{m})$ & $0.08 \pm 0.49$ & $0.49 \pm 0.25$ & $* *$ & -4.16 \\
Ashutle $\left.^{\circ}\right)$ & $14.8 \pm 8.0$ & $3.7 \pm 5.2$ & $* *$ & 6.60 \\
\hline
\end{tabular}

* Significant difference between $S G(n=14)$ and NG $(n=10)$ at $p<0.05$ level.

** Significant difference between SG $(n=14)$ and NG $(n=10)$ at $p<0.01$ level.

Table 2

Kinematic Data in the Movement Parameters

\begin{tabular}{|c|c|c|c|c|c|c|}
\hline & & & SG & NG & $p$ & $\mathrm{t}$ \\
\hline \multicolumn{3}{|c|}{$X$-factor $\left({ }^{\circ}\right)$} & $46.9 \pm 11.2$ & $36.7 \pm 8.2$ & $* *$ & 3.09 \\
\hline \multirow{5}{*}{$\mathrm{ROM}$} & & Flex/Ext $\left({ }^{\circ}\right)$ & $25.8 \pm 16.1$ & $20.9 \pm 12.9$ & ns & 1.00 \\
\hline & Shoulder & $\operatorname{Abd} / \operatorname{Add}\left({ }^{\circ}\right)$ & $14.6 \pm 6.0$ & $13.8 \pm 6.4$ & ns & 0.13 \\
\hline & & Rotation $\left(^{\circ}\right)$ & $107.5 \pm 30.9$ & $47.7 \pm 20.4$ & $* *$ & 6.85 \\
\hline & Elbow & Flex/Ext $\left({ }^{\circ}\right)$ & $70.6 \pm 9.1$ & $54.0 \pm 22.9$ & $* *$ & 2.73 \\
\hline & Wrist & Flex/Ext $\left({ }^{\circ}\right)$ & $85.9 \pm 50.4$ & $31.7 \pm 23.7$ & $* *$ & 4.18 \\
\hline \multicolumn{3}{|c|}{ PM length change (\% rest length) } & $41.3 \pm 10.9$ & $29.2 \pm 10.9$ & $* *$ & 3.22 \\
\hline
\end{tabular}




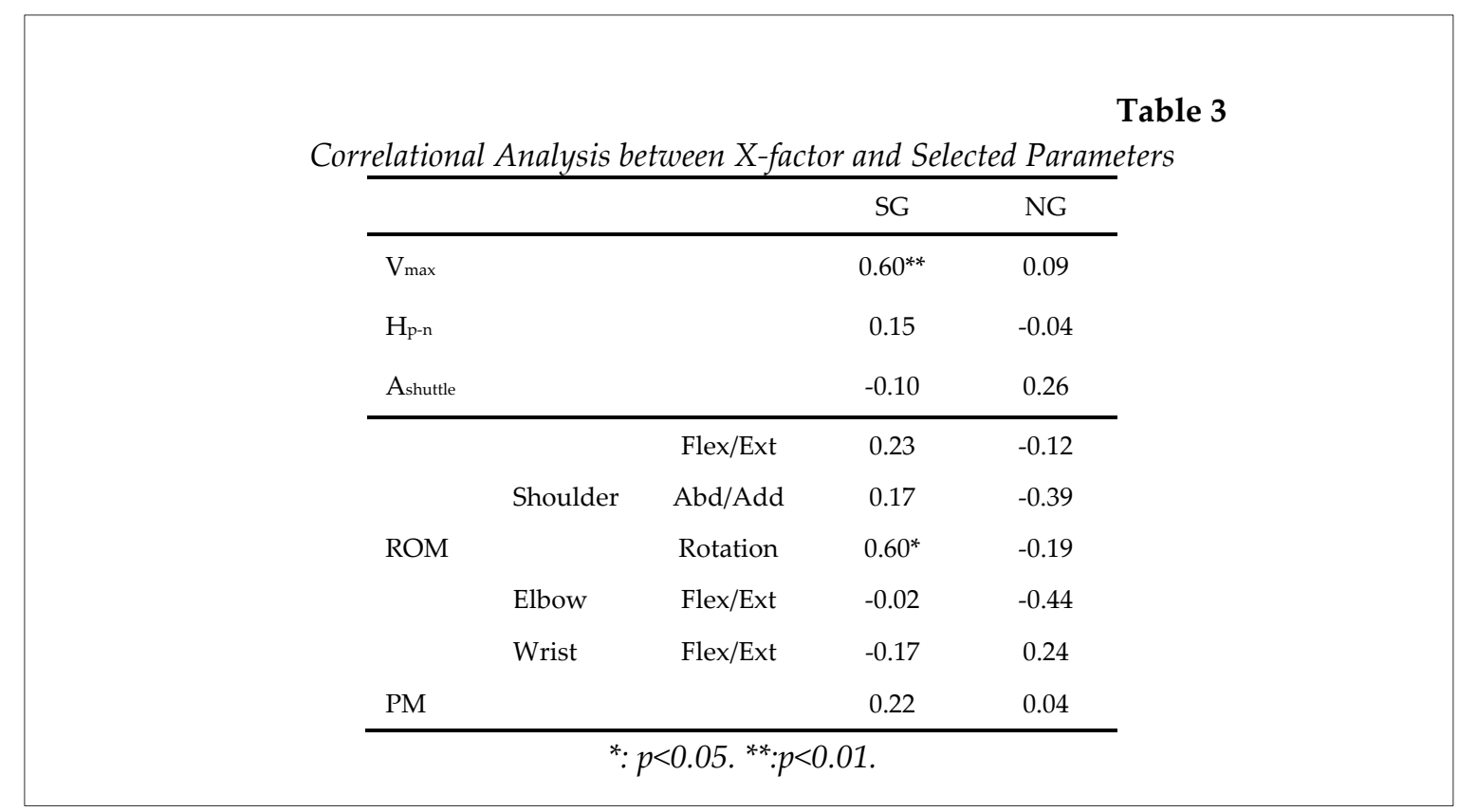

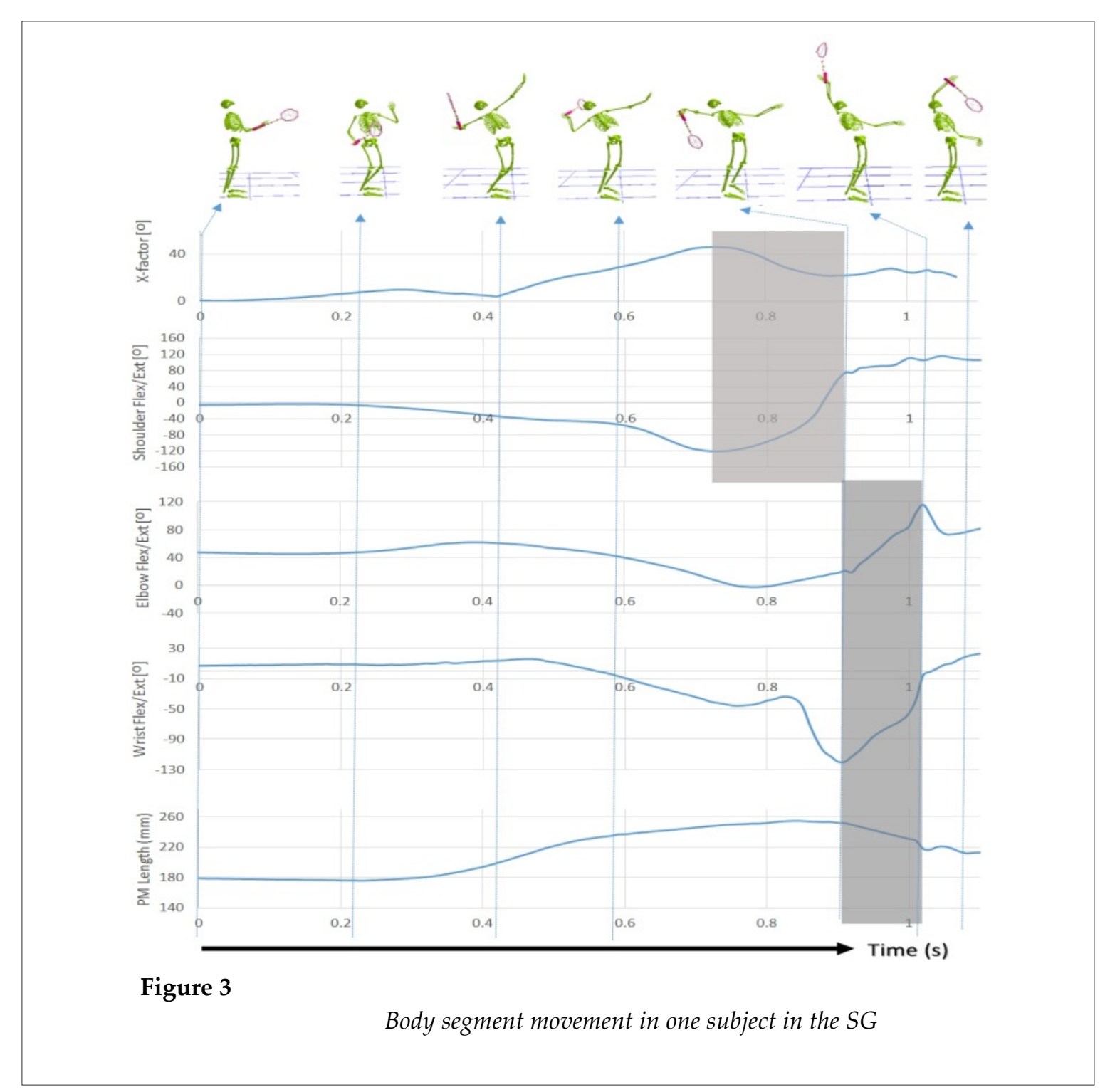




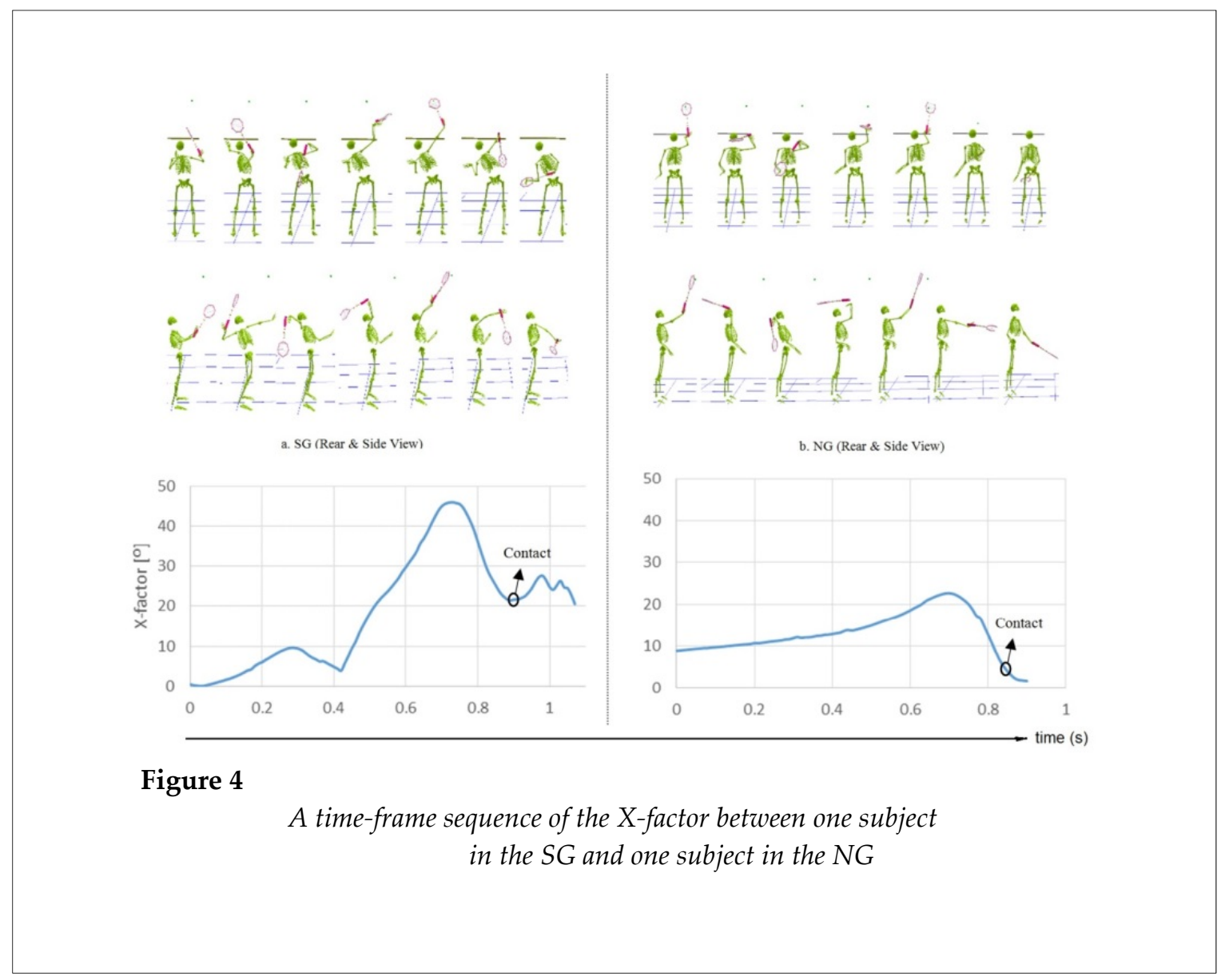

Table 3 shows a correlational analysis between the X-factor and selected parameters. A significant positive correlation was found in $\operatorname{Vmax}(p<0.01)$ and shoulder rotation $(p<0.05)$ of the SG. Negative correlations were found as follows: 1) for the SG, in the Ashuttle and elbow (flexion/extension) and wrist (flexion/extension), and 2) for the NG, in the Hp-n, shoulder (all three components - flexion/extenson, ab/adduction and rotation), and elbow (flexion/extension).

Figure 3 shows a sequential use of different body segments, typical among the SG, for the forehand smash. Forehand smash characteristics may be described as follows:

1) The duration, from the beginning of preparation to the end of the follow-through lasts about one second.

2) The preparation phase takes about three quarters of this time, during which the X-Factor gradually increases to its maximum and the shoulder reaches the point of greatest extension (minimum value). During this period, the elbow and wrist engage in extension, but only gradually and not yet to their fullest extent, while the PM has almost achieved its maximum length.

3) At this point, the acceleration phase begins, first through a fast decrease in the $X$-Factor and flexion of the shoulder, during which the elbow and wrist continue to engage in extension and the PM lengthens marginally (effectively creating a timing lag between these parameter pairs).

4) After the $X$-factor and shoulder flexion have completed their acceleration phase, the elbow and wrist begin rapid flexion and the PM starts to shorten.

Impact of the racket with the shuttlecock occurs at the point of greatest angular acceleration in both wrist and elbow flexion (the place where the slopes of the respective graphs are steepest).

\section{Discussion}

The purposes of the current study were to: 1) quantitatively describe kinematic characteristics of the forehand smash using a 15segment, full-body biomechanical model, 2) 
examine and compare kinematic differences between novice and skilled players with a focus on trunk rotation (the X-factor), and 3) through this comparison, identify principal parameters that contributed to the quality of the skill. Together, these findings have the potential to assist coaches and players in the teaching and learning of the forehand smash.

In badminton, winning points is central to the premise of game-play. One effective way of winning points is to make it difficult for one's opponent to successfully return a volley. The forehand smash has several qualities that ideally suit this objective. Three parameters in particular can be used to evaluate the effectiveness (quality) of a forehand smash: 1) the release speed of the shuttlecock (Vmax), 2) the vertical distance between the shuttlecock and the net, as the shuttlecock passes the net (Hp-n), and 3) the downward angle of the shuttlecock's flight immediately after impact from the racket (Ashuttle). The higher the shuttlecock's speed, the greater its downward angle, and the closer its clearance height to the net, the more difficult it is to return.

In the current study, the high correlation found between the X-factor and the Vrelease supports the results of Bahamonde (1999) and Teu et al. (2005), which indicated that trunk rotation contributed to more than $50 \%$ of a racket head's forward velocity while the other proximal joint actions (shoulder internal rotation) and distal joint actions (elbow extension and forearm pronation) served to position and move the arm in the general direction of the target. A comparison of the SG with the NG substantiates and adds to the above findings. With regard to Vmax, the SG generated shuttlecock speeds more than 30\% higher than the NG did. In terms of shoulder rotation, there was a high correlation with the Xfactor, but only among the SG. Shoulder rotation (i.e. supination, or external rotation) primarily generates and stores storage elastic energy during the backswing, through the coordination of adducting the shoulder and extending the elbow to reach a large $X$-factor. In examining the Hp-n and the Ashuttle, the current study reveals additional pertinent details. The average Hp-n for the SG was only $0.08 \mathrm{~m}$, whereas it was $0.49 \mathrm{~m}$ for the NG and the downward flight angle of the shuttlecock (Ashuttle) was more than four times steeper for the SG than for the NG. These last two findings may be attributed to significantly greater wrist control (ROM) among the SG (almost triple that of the NG). Collectively, these results suggest that the SG of the current study can be used as a paradigm for revealing underlying motor control mechanisms of a high quality forehand smash. In the current study, kinematic analysis revealed that skilled players utilized: 1) more trunk rotation $(28 \%), 2)$ more ROM in shoulder rotation (more than double), 3) more ROM in both elbow and wrist flexion/extension (more than $30 \%$ and $170 \%$, respectively), and 4) more PM lengthening $(38 \%)$ than novices. Correlation analysis showed trunk rotation (the $\mathrm{X}$-factor) to be integral for maximizing the release speed of the shuttlecock and a notable contributor to skill effectiveness. This confirms results found in studies of other sports, for example the golf swing (Egret et al., 2004). As a matter of note, the current study established the X-Factor to be $46.9 \pm 11.2^{\circ}$, whereas Egret et al. (2004) demonstrated that expert and experienced golfers achieved an $\mathrm{X}$ factor of $47.7^{\circ}$ and $46.2^{\circ}$ respectively. The similarity in these findings can be explained in two ways: 1) trunk rotation is vital in the acceleration of equipment prior to striking the intended object, and 2) $46-48^{\circ}$ appears to be approaching the practical physiological limits for trunk rotation. Further studies are needed to confirm this hypothesis. The X-Factor is present in the NG $\left(36.7 \pm 8.2^{\circ}\right)$, but it is significantly smaller than for the SG. In the NG, correlation analysis revealed the $\mathrm{X}$-Factor not to play any significant role in generating shuttlecock speed. It is possible that this is a 'natural' response to the reaching aspect of the task that was involved in this test. Collectively, this suggests that the X-Factor has an operational range wherein it contributes optimally to shuttlecock velocity. For coaches, this has important ramifications; the benefits of trunk rotation are only achieved when it can be purposefully trained.

Based on results from the SG, it can be shown that a high quality forehand smash is a consequence of a sequentially unfolding series of segmental/joint control. In the simplest terms, the movement can be understood as having three phases: preparation, acceleration and followthrough. The reality is more nuanced; the forehand smash can best be understood by 
examining the changes in slope of the graphed elements in Figure 3. The places where graph slopes are steepest are where the individual elements make their greatest contributions to acceleration of the racket.

Looking at Figure 3, each of the parameters examined has a discrete control contribution to make to the forehand smash, and each clearly begins and ends its respective contribution at a different time. Preparation of a forehand smash begins as the $\mathrm{X}$-factor starts to increase (trunk rotation toward the racket-hand side). This is followed first by an increase in shoulder extension (a backward reaching movement) then, almost simultaneously, elbow flexion/extension and PM lengthening, and finally the start of wrist flexion/extension.

The end of the preparation phase and the beginning of the acceleration phase occur as the $\mathrm{X}$-Factor and shoulder flexion/extension reach their most extreme positions and begin to reverse their preparatory movements - the trunk starts to rotate toward the non-racket-hand side and the shoulder begins flexion. The elbow ends its preparatory movement shortly thereafter and begins its contribution to the acceleration through the commencement of extension. The wrist and $\mathrm{PM}$ only begin acceleration as the X-factor and shoulder flexion have finished theirs. The acceleration phase ends at the striking of the shuttlecock. The features that demark this point are: 1) elbow extension and wrist flexion achieve maximum velocity/acceleration simultaneously, 2) elbow extension ends, 3) there is significant slowing of wrist flexion, and 4) there is a noticeable change in the speed of PM shortening. The simultaneity of segmental motor behaviors at this point suggests that the rapid contraction of the PM prior to striking the shuttlecock contributes, in greatest part, to the internal rotation of the upper arm, forearm pronation and wrist flexion. More in-depth studies will be required to confirm this and the contributions of other muscle groups to the forehand smash.

Collectively, the sequential and/or overlapping motor control described above is the means by which expert players generate high racket velocities. It results in a proximal to distal movement pattern that can best be described as whip-like. The greatest advantage of this control pattern is that it maximizes the angular momentum of the distal segment by transferring momentum consecutively from larger to smaller body segments (Shan et al., 2015; Shan and Westerhoff, 2005; Zhang and Shan, 2014). Formation of an X-Factor causes lengthening in the rectus abdominus, transverse abdominus, the internal \& external obliques, the PM, the elbow flexors and the shoulder extensors. As revealed by Gowitzke and Milner (1980), prelengthening of 120 to $130 \%$ (measured from resting length) led to maximum muscle tension. This kind of dynamic muscle pre-lengthening was also reported in a kicking study (Shan and Westerhoff, 2005). In this case, trunk flexors, hip flexors and quadriceps engaged in lengthening which helped generate larger muscle forces and increase effectiveness of the kicking action.

Figure 4 allows easy visual comparison of the X-Factor using trials that can be considered representative of the SG and the NG. The larger rotational potential for the SG allows higher shuttlecock velocities to be achieved. Besides having larger rotational angles for the X-Factor, a second, much smaller, increase in the $\mathrm{X}$-factor is present for the SG in the follow-through phase of the stroke. The typical NG exhibits no such characteristics, with the shuttlecock strike occurring as the X-Factor is still in its rotational movement toward the non-racket-hand side. The difference in this case can perhaps be taken as a measure of expertise. The SG employed a high degree of automatic action and highly effective body control. The SG directed energy toward both power and control of the strike, whereas the NG seemed to be concerned with generating power. Postural control observed among the SG could suggest players to be maintaining a heightened state of readiness for game-play; an effect that is the result of long-term training. The NG had lesser effective body control and coordination as they were only in the first stages of learning the skill.

The current study is the first to employ 3D, full-body modeling to examine a badminton skill - the forehand smash. Prior to the current study, research employing two-dimensional (e.g. video analysis) or partial-body models (e.g. upper limb) were not able to fully explore the interaction between the X-Factor and the joint coordination in the kinematic chain governing the stroke. By including quantitative data from the movements 
of the trunk, results from the current study can be used to better understand fundamental factors influencing the quality of skill execution.

The current study has several limitations. First, data was gathered in a laboratory environment to control as many confounding variables as possible. Second, a larger sample size would allow a more comprehensive analysis. In future studies, recruiting and testing of more professional badminton players will be necessary to investigate long-term training effects. Third, due to a limited pool of subjects (14 SG, $10 \mathrm{NG}$ ), it was not viable to explore the influence of gender. This should also be considered in further research. Finally, future studies might investigate actual game-play in order to reveal the contribution of the lower extremity to the forehand smash.

\section{Conclusion}

In conclusion, previous studies of the forehand smash have failed to examine full-body contributions to the quality of a player's stroke, especially the influence of the trunk segment. In the current study, skilled players demonstrated greater use of trunk rotation (X-Factor) to initiate sequential, proximal to distal, segmental control in order to maximize the angular momentum of the racket head at the point of impact with the shuttlecock. This whip-like control pattern was not observed among novices. Control and quality of the stroke were also different among the two test groups (SG \& NG). Quality of the forehand stroke was determined by examining three parameters: Shuttlecock Maximum Flight Velocity (Vmax), Past-Net Height (Hp-n), and Shuttlecock Flight Angle (Ashuttle). Due to their use of greater trunk rotation and pre-lengthening of the pectoralis major, the SG was able to generate higher shuttlecock velocities. Control of the Hp-n and the Ashuttle was significantly better among the SG than the NG, a finding that may be explained by their greater use of wrist flexion (almost triple). In combination, the findings of the current study can provide valuable information for training practices intended to increase the efficacy of this skill. Indeed, the current study underscores the need for more 3D, full-body analyses of badminton skills.

\section{Acknowledgements}

This work was supported by Sport Science Association of Alberta (SSAA/Canada) and National Sciences and Engineering Research Council of Canada (NSERC). The authors wish to thank all the participants who donated their time and expertise.

\section{References}

Adrian MJ, Enberg M. Sequential timing of three overhand patterns. In Kinesiology Review. Washington, D.C.: The Council on Kinesiology of the Physical Education Division, 1- 9; 1971

Aguinaldo AL, Buttermore J, Chambers H. Effects of upper trunk rotation on shoulder joint torque among baseball pitchers of various levels. J Appl Biomech, 2007; 23: 42-51

Bahamonde R. Producing an explosive forehand and backhand. In Applied Proceedings of the XVII International Symposium on Biomechanics in Sports. Australia: International on Biomechanics of Sports, 17-25; 1999

Brahms BV. Badminton Handbook. Maidenhead: Meyer \& Meyer Verlag Sport (UK) Ltd; 2014

Brundle FF. Badminton. London: Acro Publications; 1963

Cheetham PJ, Martin PE, Mottram R, St Laurent B. The importance of stretching the "X-Factor" in the downswing of golf: The "X-Factor Stretch." In Optimising Performance in Golf. Brisbane, Australia: Australian Academic Press, 192-199; 2001

Chu Y, Sell TC, Lephart SM. The relationship between biomechanical variables and driving performance during the golf swing. J Sports Sci, 2010; 28: 1251-1259

Dapena J. A method to determine the angular momentum of a human body about three orthogonal axes passing through its center of gravity. J Biomech, 1978; 11: 251-256

Dixon WJ, Massey FJ. Introduction to statistical analysis. New York: McGraw-Hill; 1969

Egret C, Dujardin F, Weber J, Chollet D. 3D kinematic analysis of the golf swings of expert and experienced golfers. J Hum Movement Stud, 2004; 47: 193-204 
El-Gizawy H, Akl AR. Relationship between reaction time and deception type during smash in badminton. J Sport Res, 2014; 1: 49-56

Ellenbecker TS, Davies GJ. Closed kinetic chain exercise: A comprehensive guide to multiple joint exercise. Palmer: Human Kinetics Publisher; 2001

Ellenbecker TS, Roetert EP. An isokinetic profile of trunk rotation strength in elite tennis players. Med Sci Sport Exer, 2004; 36: 1959-1963

Elliott B. Hitting and kicking. In Biomechanics in sport: Performance Enhancement and Injury Prevention. Oxford: Blackwell, 487-504; 2000

Elliott B, Marsh A, Overheu P. The topspin backhand drive in tennis-A biomechanical analysis. J Hum Movement Stud, 1989; 16: 1-16

Gowitzke B, Waddell D. Biomechanical studies of badminton overhead power strokes: A review. In Biomechanics in Sports IX. Ames, IA: International Society of Biomechanics in Sport, 267-272; 1991

Gowitzke BA, Milner M. Understanding the scientific basis of human movement. Baltimore: Williams and Wilkins Co; 1980

Hamilton N, Weimar W, Luttgens K. Kinesiology: Scientific basis of human motion. London: McGraw-Hill Humanities/Social Sciences/Language; 2002

Lawrence I, Lin K. A concordance correlation coefficient to evaluate reproducibility. Biometrics, 1989; 45: 255268

Li S, Zhang Z, Wan B, Wilde B, Shan G. The Relevance of Body Positioning and Its Training Effect on Badminton Smash. J Sport Sci, 2016, DOI: 10.1080/02640414.2016.1164332. Available at: http://www.tandfonline.com/doi/abs/10.1080/02640414.2016.1164332; accessed on 16.05.2016

Lo D, Stark K. Sports performance series: The badminton overhead shot. J Strength Cond Res, 1991; 13: 6-15

McTeigue M, Lamb SR, Mottram R. Spine and hip motion analysis during the golf swing. In Science and golf II. London: Taylor \& Francis, 50-57; 1994

Meister DW, Ladd AL, Butler EE, Zhao B, Rogers AP, Ray CJ, Rose J. Rotational biomechanics of the elite golf swing: Benchmarks for amateurs. J Appl Biomech, 2011; 27: 242-251

Ming CS. Badminton wall practice and training: A practical approach. J Phys Educ, Recreation \& Dance, 1993; 64: $17-20$

Osiński W. Anthropomotorics. Poland: WF Poznań; 2003

Phomsoupha M, Laffaye G. The science of badminton: game characteristics, anthropometry, physiology, visual fitness and biomechanics. Sports Med, 2015; 45(4): 473-495

Putnam CA. Sequential motions of body segments in striking and throwing skills: Descriptions and explanations. J Biomech, 1993; 26: 125-135

Rantzmayer J. Wrist snap - myth or reality? Badminton Gazette, 1977; 58: 128-129

Roetert P, Groppel J. World-class tennis technique. England: Human Kinetics Publisher; 2001

Sakurai S, Ohtsuki T. Muscle activity and accuracy of performance of the smash stroke in badminton with reference to skill and practice. J Sports Sci, 2000; 18: 901-914

Seroyer S, Nho S, Bach B, Bush-Joseph C, Nicholson G, Romeo A. The Kinetic Chain in Overhand Pitching. Sports Health, 2010; 2(2): 135-146

Shan G, Bohn C. Anthropometrical data and coefficients of regression related to gender and race. Appl Ergon, 2003; 34(4): 327-337

Shan G, Bohn C, Sust M, Nicol K. How can dynamic rigid-body modeling be helpful in motor learning? Learning performance through dynamic modeling. Kinesiology, 2004; 36: 182-191

Shan G, Westerhoff P. Full-body kinematic characteristics of the maximal instep soccer kick by male soccer players and parameters related to kick quality. Sports Biomech, 2005; 4: 59-72

Shan G, Visentin P, Zhang X, Hao W, Yu D. Bicycle kick in soccer: is the virtuosity systematically entrainable? Sci Bull, 2015; 60: 819-821

Shan G, Zhang X, Li X, Hao W, Witte K. Quantification of Golfer-club Interaction and Club-type's Affect on Dynamic Balance during a Golf Swing. Int J Perform Anal Sport, 2011; 11(3): 417-426

Stigler SM. Francis Galton's account of the invention of correlation. Stat Sci, 1989; 4: 73-79

Tang H, Abe K, Katoh K, Ae M. Three-dimensional cinematographical analysis of the badminton forehand

(C) Editorial Committee of Journal of Human Kinetics 
smash: movements of the forearm and hand. In Science and Racket Sports. London: E \& FN Spon, 113120; 1995

Teu KK, Kim W, Tan J, Fuss FK. Using dual Euler angles for the analysis of arm movement during the badminton smash. Sports Eng, 2005; 8: 171-178

Wang J, Moffit J. Teaching badminton based on student skill levels. Strategies, 2009; 22: 14-18

Werner S, Guido J, Stewart G, McNeice R, VanDyke T, Jones D. Relationships between throwing mechanics and shoulder distraction in collegiate baseball pitchers. J Shoulder Elbow Surg, 2007; 16(1): 37-42

Wilkinson AT, Strkalj G, Shan G. Use of the race concept in anthropometry. Appl Ergon, 2005; 36(1): 121-122

Xinhua News, Chinese Fu Clocks Fastest Smash at Sudirman Cup - Recent Findings, 2005. Available at: http://china.org.cn/english/sports/128755.htm; accessed on 22.05.2015

Yap C. Badminton- Fastest racket sport. Badminton Information - Recent Findings, 2012. Available at: http://www.badminton-information.com; accessed on 17.05.2015

Zhang $X$, Shan G. Where do golf driver swings go wrong? Factors influencing driver swing consistency. J Med Sci Sports, 2014; 24: 749-757

\section{Corresponding author:}

\section{Dr. Gongbing Shan, Professor}

Director of Biomechanics Lab

Department of Kinesiology, University of Lethbridge

4401 University Drive

Lethbridge, AB, Canada, T1K 3M4

Phone: 1-403-3292683;

Fax : 1-403-3801839;

E-mail: g.shan@uleth.ca 